\title{
Clinical potential of boron neutron capture therapy for locally recurrent inoperable previously irradiated head and neck cancer
}

\author{
Diana Lim ${ }^{\mathrm{a}}$, Daniel SC Quah ${ }^{\mathrm{b}}$, Michelle Leech $^{\mathrm{a}}$, Laure Marignol ${ }^{\mathrm{a}, *}$ \\ a Applied Radiation Therapy Trinity, Discipline of Radiation Therapy, School of Medicine, Trinity Centre for Health sciences, Trinity College Dublin, Ireland \\ ${ }^{\mathrm{b}}$ Department of Radiation Oncology, National Cancer Centre, Singapore
}

\section{H I G H L I G H T S}

- BNCT can prolong median overall survival.

- BNCT can be associated with severe adverse effects.

- BNCT may be comparable to chemotherapy-based regimens.

- BNCT may be comparable to re-irradiation techniques regimens in patients with low performance status.

\section{A R T I C L E I N F O}

\section{Article history:}

Received 19 January 2015

Received in revised form

23 June 2015

Accepted 25 July 2015

\begin{abstract}
A B S T R A C T
This review compares the safety and efficacy of boron neutron capture therapy (BNCT) in the treatment of previously irradiated, inoperable locoregional recurrent HNC patients and compares BNCT against the standard treatment of platinum-based chemotherapy. Our analysis of published clinical trials highlights efficacy of BNCT associated with mild side effects. However, the use of BNCT should be explored in stratified randomised trials.
\end{abstract}

(c) 2015 Published by Elsevier Ltd.

\section{Introduction}

Patients with Locally Recurrent Head and Neck Cancer (LRHNC) have limited treatment options (Vermorken and Specenier, 2010). Optimal therapy has not been established and prognosis is generally poor (Tanvetyanon et al., 2009). Combination chemotherapy has been used, but results are far from satisfactory. The addition of EGFR inhibitors (cetuximab) conferred a median survival of 10.1 months, more than the 7.4 months of those who received chemotherapy only (EXTREME trial) (Vermorken et al., 2008; Vermorken and Specenier, 2010). Re-irradiation utilizing alternate techniques that can deliver a high tumouricidal dose while limiting normal tissue exposure has potential, and in this context, Boron Neutron Capture Therapy (BNCT) has also been explored. The first registered Phase II trial addressing the use of BNCT in Head and Neck Cancer (Kankaanranta et al., 2007, 2012) showed that it is effective in managing patients with LRHNC, but to date no studies have compared its potential to systemic therapy. This review aims to assess the clinical potential of BNCT in comparison to

\footnotetext{
* Corresponding author.

E-mail address: marignol@tcd.ie (L. Marignol).
}

systemic therapies alone, systemic and radiation therapies and radiation therapy alone and thereby make observations on its potential for the improved management of these patients.

\section{Materials and methods}

Published BNCT studies were identified on MEDLINE using the following key words: "Head and Neck Neoplasms" [Mesh] AND "Neoplasm Recurrence, Local/radiotherapy" [Mesh] AND "Boron Neutron Capture Therapy"[Mesh]. RCTs were identified using the following MEDLINE search strategy: "Head and Neck Neoplasms" [Mesh] AND "drug therapy"[Subheading]) AND "Cisplatin/administration and dosage" [Mesh] AND "Neoplasm Recurrence, Local/ drug therapy" [Mesh] AND "Clinical Trial, Phase III" [Publication Type]. The Cochrane Library was searched using the following key words: "head and neck neoplasm" AND chemotherapy NOT radiotherapy. Clinicaltrials.gov was searched using: "head and neck neoplasm" AND chemotherapy NOT radiotherapy. Seven phase I and phase II BNCT trials reporting the use of BNCT with intravenous administration of boron-carrier in patients with previously treated loco-regionally recurrent unresectable HNC were 
identified. (Aihara et al., 2014; Ariyoshi et al., 2007; Kankaanranta et al., 2012; Kato et al., 2009; Kimura et al., 2009; Suzuki et al., 2014; Wang et al., 2011). The EXTREME phase III randomized controlled trial evaluating clinical outcomes of standard systemic therapy in this same category of patients was included to assess standard therapy (Vermorken and Specenier, 2010). The primary outcomes used to evaluate the safety and efficacy of BNCT for treatment of locally recurrent inoperable HNC patients were response rates (Complete and Partial Response), survival and incidence of severe acute adverse events (National Cancer Institute Common Terminology Criteria for Adverse Events version 3.0 ( $\mathrm{NCI}$ CTC) Grade 3-5). The secondary outcomes considered to compare BNCT to systemic therapies were response rate, survival and incidences of severe acute adverse events ( $\mathrm{NCI}$ CTC version 3.0 Grade 3-5). The descriptions of original studies were assessed by using frequency, 95\% confidence intervals and forest plots. Statistical heterogeneity across trials was assessed using the chi-squared $\left(X^{2}\right)$ test and consistency between studies with the $I^{2}$ statistic (Higgins and Thompson, 2002). Fishers' exact tests were used to compare groups with respect to dichotomous end points (eg, response rates and toxicities). A t-test was performed according to the methodology described in (Hozo et al., 2005) to compare reported survival data between BNCT vs CTX, and between BNCT vs CTX/C225. All $P$ values reported are two-sided. $P<0.05$ was considered statistically significant.

\section{Results}

\subsection{Efficacy and safety of BNCT}

Median overall survival of 13.1 and 9.7 months were reported (Kankaanranta et al., 2012; Suzuki et al., 2014). A survival time of up to 72 months was reported for one patient after receiving seven BNCT treatments over 6 years (Kato et al., 2009). Reports of progression-free survival ranged from 5.1 to 7.9 months (Kankaanranta et al., 2012; Suzuki et al., 2014). Reported response rates, based on the Response Evaluation Criteria for solid tumours, ranged from $61 \%$ to $100 \%$. The heterogeneity of the treatment response between trials was significant $\left(X^{2}(5, N=115)=4.878\right.$, $p<0.05)$. There was no evidence of statistical heterogeneity between trials $\left(I^{2}=17.9 \%, p=0.3\right)$. The weighted frequency of the response rate of these 5 trials was $72.1 \%$ (95\%CI: [62.5-78.8]). All BNCT trials evaluated toxicity and severe adverse effects (Fig. 1). The incidence of Grade 3 toxicities was up to $53 \%$, while that of Grade 4 was below 10\%. Three treatment-related deaths were reported in a single trial (Suzuki et al., 2014). Mucositis was the most commonly reported toxicity.

\subsection{BNCT vs standard chemotherapy}

The overlay of the Kaplan-Meier curves from the two arms of the EXTREME trial (Vermorken and Specenier, 2010) and the Kankaanranta BNCT study (Kankaanranta et al., 2012) suggests that a proportion of patient treated with BNCT exhibit a better outcome than those receiving platinum chemotherapy alone or with combination of Cetuximab (Figs. 2 and 3). All 30 patients evaluated in the BNCT trial had inoperable head-and-neck cancers that had recurred locally with/without metastasis after surgery and prior conventional radiotherapy or chemoradiation therapy. The 442 patients included in the EXTREME trial had recurrent and/ or metastatic squamous-cell carcinoma of the head and neck that were ineligible for local therapy. Patients from all groups received some form of treatment before disease recurrence and/or metastases. 53\% of patients in the BNCT group had WHO Performance Status $>1$, similar to Karnofsky score $($ KPS $)<80$, in contrast to $11 \%$ and $12 \%$ of the CTX and the CTX $+\mathrm{C} 225$ group respectively. The median survival times for all patients offered BNCT, CTX/C225 and CTX were 13.1 months, 10.1 months and 7.4 months respectively (Tables 1 and 2). The survival advantage when compared to CTX/ C225 and CTX were both considered statistically significant $(p<0.0001)$. The hazard ratio (HR) was $\sim 0.78$ (95\% CI: $0.5,1.49)$ for BNCT vs CTX/C225 and $~ 0.57$ (95\% CI: $0.36,0.9)$ for BNCT vs CTX. The percentage of patients who were alive at 1 year was higher for patients who received BNCT as compared to both CTX/ C225 and CTX (60\% vs 37\% vs 29.5\%). Long term survival ( $\geq 2$ years) was also increased for patients treated with BNCT (30\% vs $<1 \%$ ) and $18 \%$ of the patients treated with BNCT were still alive after 4 years. PFS was significantly improved in patients treated with BNCT than those receiving CTX/C225 and CTX $(p<0.0001)$ The response rates for BNCT appear to be significantly better when compared to either arm of the EXTREME trial (both arms, $p=0.001$ ) (Table 1 ). While the percentages of Grade 3 toxicities were highest in the BNCT group (53\%) and lowest in the CTX group (45\%), Grade 4 side effects were significantly reduced in the BNCT group (3\%) as compared to $31 \%$ of each of the CTX and CTX/C225 trials. The Grade 3 and 4 adverse toxicity profile for BNCT was significantly better than CTX $(p=0.0444)$ and CTX/C225 $(p=0.0032)$.

Median OS was measured from the time of randomization in the phase III EXTREME study but from the time of first treatment in the BNCT study. Time from randomization to treatment

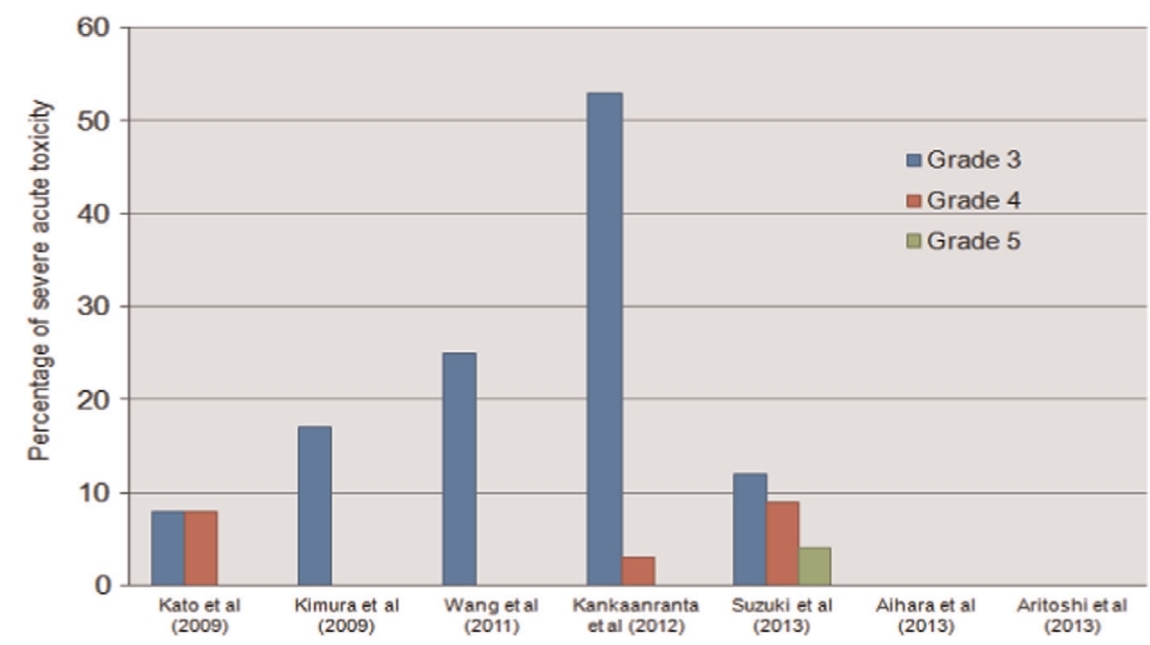

Fig. 1. Incidence (\%) of reported severe acute adverse events (Grade 3-5) across BNCT trials. 


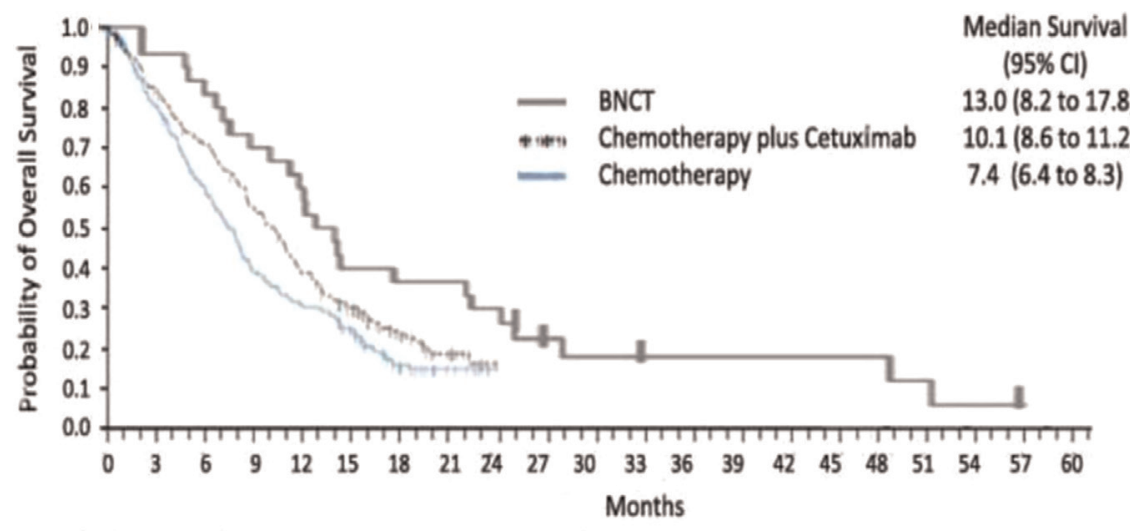

$\begin{array}{lrllll}\begin{array}{l}\text { Number at risk } \\ \text { BNCT }\end{array} & 30 & & & & 9 \\ \text { Chemotherapy } & 220 & 127 & 65 & 19 & 1 \\ \begin{array}{c}\text { Chemotherapy } \\ + \text { Cetuximab }\end{array} & 222 & 153 & 82 & 30 & 3\end{array}$

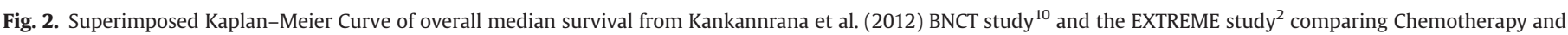
Chemotherapy with Cetuximab.

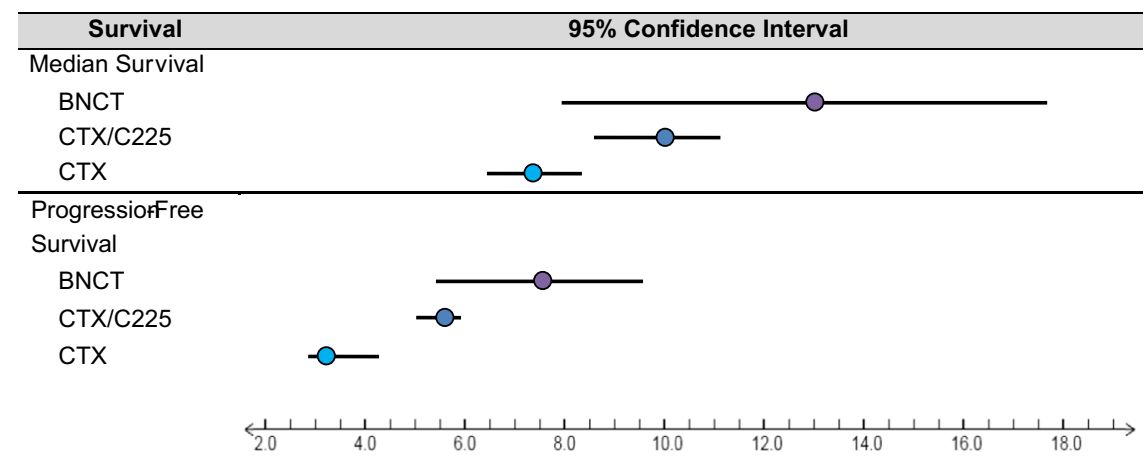

Fig. 3. Comparison of Published confidence intervals from BNCT study and both arms of EXTREME study.

Table 1

Baseline patient characteristics for patients treated with Boron Neutron Capture Therapy (BNCT), Platinum-based Chemotherapy with Cetuximab (CTX/C225), Platinum-based Chemotherapy only (CTX).

\begin{tabular}{|c|c|c|c|c|}
\hline Parameter & & BNCT & CTX/C225 & CTX \\
\hline Number of patients & & 30 & 222 & 220 \\
\hline Median Age (range) (years) & & $61(37-79)$ & 56 & 57 \\
\hline \multicolumn{5}{|l|}{ Previous Treatment (\%) } \\
\hline Chemotherapy & & 33 & 41 & 36 \\
\hline Radiotherapy & & 100 & 85 & 86 \\
\hline \multicolumn{5}{|l|}{ Performance status (\%) } \\
\hline Karnofsky & & & & \\
\hline \multirow[t]{2}{*}{0} & 0 & & & \\
\hline & & 47 & 88 & 89 \\
\hline$\geq 80$ & 47 & & & \\
\hline 2 & 43 & 53 & 12 & 11 \\
\hline$<80$ & 10 & & & \\
\hline
\end{tabular}

KPS, Karnofsky Performance Status; ECOG, Eastern Cooperative Oncology Group. Relationship between ECOG and Karnofsky performance status are adopted from study by Oken et al. (1982).

commencement was not reported in the EXTREME trial. Similarly, PFS for the EXTREME study was reported from the time of randomization whereas the BNCT trial measured it from the time of first BNCT until the disease progressed locally or distally or death, whichever came first. The EXTREME Study used the Modified World Health Organization criteria to determine tumor response and disease progression. Tumour response was evaluated at 6 -week intervals. In the BNCT study, treatment response was evaluated by use of the RECIST (Response Evaluation Criteria in
Table 2

Summary of patient survival parameters and record acute adverse events in patients receiving Boron Neutron Capture Therapy (BNCT), Platinum-based Chemotherapy with Cetuximab (CTX/C225), Platinum-based Chemotherapy only (CTX).

\begin{tabular}{llll}
\hline Parameter & BNCT (95\%CI) & CTX/C225 (95\%CI) & CTX (95\%CI) \\
\hline $\begin{array}{lll}\text { Median OS (months) } \\
\quad \text { All patients }\end{array}$ & $13.0(8.2-17.8)$ & $10.1(8.6-11.2)$ & $7.4(6.4-8.3)$ \\
Median PFS (months) & & & \\
$\quad$ All patients & $7.5(5.4-9.6)$ & $5.6(5.0-6.0)$ & $3.3(2.9-4.3)$ \\
Response Rate (\%) & $76(61-91)$ & $36(29-42)$ & $20(15-25)$ \\
$\quad$ Complete Response & $45(27-63)$ & - & - \\
$\quad$ Partial Response & $31(14-48)$ & - & - \\
Acute adverse events & & & 45 \\
Grade 3 (\%) & 53 & 51 & 31 \\
$\quad$ Grade 4 (\%) & 3 & 31 & 3 \\
Grade 5 (\%) & 0 & 1 &
\end{tabular}

OS, Overall Survival; PFS, Progression-Free Survival; KPS, Karnofsky Performance Status; CI, Confidence interval.

Solid Tumors), performed at 1, 3, 6 and 12 months after neutron irradiation. Adverse effects were evaluated according to the $\mathrm{Na}$ tional Cancer Institute Common Terminology Criteria (NCI-CTC) in both papers.

\section{Discussion}

It is believed that LRHNC tumors represent clonogens that have been exposed to selective pressures during initial RT that cause 
radioresistance (Weichselbaum et al., 1988), and to control these recurrences, a greater tumoricidal dose may be required. Re-irradiation of critical normal tissues is difficult as these tissues are already close to the assumed lifetime tolerance doses following prior irradiation. Treatment approaches that allow dose escalation for tumor control and can effectively spare normal tissue during re-irradiation would be the ideal treatment for this group of patients. Boron Neutron Capture Therapy is a form of treatment that can address these issues. BNCT, being a single day treatment, can potentially offer these patients treatment-free improvement in overall survival. The clinical potential of BNCT has been reported in several solid tumours, including Glioblastoma Multiforme (GBM), recurrent head and neck cancer, malignant melanoma and hepatocellular carcinoma (reviewed in (Barth and Joensuu, 2007)).

A median survival of 9.7 months and 13.1 months for BNCTtreated patients with recurrent HNC was reported in two out of the 7 eligible trials (Kankaanranta et al., 2012; Suzuki et al., 2014). This is an improvement from an estimated lifespan of 6 months had these patients been offered supportive care only (Zafereo et al., 2009). Median survival could not be reported in the other 5 trials because a large proportion of the patients were still alive at the study end-point. A wide range of survival assessment from as low as one to two months to as high as 72 months was reported (Kato et al., 2009). However, analysis of survival data was further complicated by a lack of standardisation in the reporting of outcomes. Only 2 studies reported on progression-free survival (up to 7.9 months) (Kankaanranta et al., 2012; Suzuki et al., 2014). This end-point is important for these patients, as head and neck tumours may cause a loss of organ function and cosmesis issues that may impair the patients' quality of life (Zafereo et al., 2009). Generally these radioresistant recurrent head and neck tumours, responded well to BNCT with at least $71.2 \%$ of the patients experiencing tumour shrinkage of more than $30 \%$ or complete disappearance of tumour. However, despite claims of good normal tissue sparing, side effects, sometimes severe, were still noted. To overcome this problem, newer and more specific boron carriers need to be engineered, with the latest developed carriers already increasing tumour uptake from the current 2.5- to an impressive 9-fold, when compared to normal tissues (List et al., 1999).

The current standard of care for LRHNC is platinum-based combination chemotherapy, usually cisplatin or carboplatin, both alkylating agents, with 5-flurouracil, and an anti-metabolite. When the monoclonal anti-EGFR antibody Cetuximab was administered alongside concurrent platinum-based chemotherapy (CTX/C225), a survival benefit of 2.5 months was reported, when compared to platinum-based chemotherapy only (CTX) (Vermorken et al., 2008). Although patients from the BNCT trials were generally of poorer performance status, their 1 and 2-year survival were approximately higher than in patients treated with CTX or CTX/C225. Long-term survival (4 years), PFS and response rates were also superior in patients treated with BNCT. This is crucial for this group of patients as uncontrolled disease at the primary site or regional lymph nodes may cause complications and lead to development of metastatic disease (Zimmermann et al., 2006). Furthermore, no weight loss was reported after the first BNCT treatment. While the EXTREME studies included patients LRHNC and metastatic disease, approximately half of patients in each arm of the study had only locoregional disease and no significant difference in survival was reported between patients with metastatic or recurrent and metastatic disease and those with only recurrent locoregional disease. These results must also be considered in the context of trials in which radiation therapy has been re-administered to previously irradiated patients who present with LRHNC. Dornoff et al. report 1 year overall survival rates for RT with cetuximab of $44.4 \%$ and of RT with cisplatin of $45.5 \%$ in a cohort of 66 re-irradiated patients with LRHNC (Dornoff et al., 2015). Other studies have yielded similar results with reports of median progression-free survival from re-irradiation of 15.0 months (Mallick et al., 2014) to 2 year overall survival rates of 43\% (Riaz et al., 2014). Developments in modern radiation therapy such as stereotactic body radiation therapy (SBRT) delivered with concomitant cetuximab have yielded one year overall survival rates of $47.5 \%$ (Lartigau et al., 2013).

\section{Conclusion}

This review highlights the possible potential of BNCT in the management of patients with LRHNC, but BNCT should be compared against other emerging local re-irradiation therapeutic options for patients with LRHNC - Stereotactic Radiosurgery (Roh et al., 2009), IMRT (Lee et al., 2007) or Proton Therapy (Stuschke et al., 2013). While the availability of BNCT facilities remains limited, it has been estimated that accelerator-based BNCT facilities can be installed in hospitals at a similar cost to that of presently used for standard radiotherapy (Blue and Yanch, 2003). Nonetheless issues with safety associated with the delivery of neutrons will have to be addressed. Further evaluation of this treatment option is warranted.

\section{References}

Aihara, T., Morita, N., Kamitani, N., Kumada, H., Ono, K., Hiratsuka, J., Harada, T. 2014. Boron neutron capture therapy for advanced salivary gland carcinoma in head and neck. International journal of clinical oncology 19, 437-444.

Ariyoshi, Y., Miyatake, S., Kimura, Y., Shimahara, T., Kawabata, S., Nagata, K., Suzuki, M., Maruhashi, A., Ono, K., Shimahara, M., 2007. Boron neuron capture therapy using epithermal neutrons for recurrent cancer in the oral cavity and cervical lymph node metastasis. Oncol. Rep. 18, 861-866.

Barth, R.F., Joensuu, H., 2007. Boron neutron capture therapy for the treatment of glioblastomas and extracranial tumours: as effective, more effective or less effective than photon irradiation? Radiother. Oncol. 82, 119-122.

Blue, T.E., Yanch, J.C., 2003. Accelerator-based epithermal neutron sources for boron neutron capture therapy of brain tumors. J. Neuro-Oncol. 62, 19-31.

Dornoff, N., Weiss, C., Rodel, F., Wagenblast, J., Ghanaati, S., Atefeh, N., Rodel, C., Balermpas, P., 2015. Re-irradiation with cetuximab or cisplatin-based chemotherapy for recurrent squamous cell carcinoma of the head and neck. Strahlentherapie and Onkologie 191, 656-664.

Higgins, J.P., Thompson, S.G., 2002. Quantifying heterogeneity in a meta-analysis. Stat. Med. 21, 1539-1558.

Hozo, S., Djulbegovic, B., Hozo, I., 2005. Estimating the mean and variance from the median, range, and the size of a sample. BMC Med. Res. Methodol. 5, 1-10.

Kankaanranta, L., Seppala, T., Koivunoro, H., Saarilahti, K., Atula, T., Collan, J., Salli, E. Kortesniemi, M., Uusi-Simola, J., Makitie, A., Seppanen, M., Minn, H., Kotiluoto, P., Auterinen, I., Savolainen, S., Kouri, M., Joensuu, H., 2007. Boron neutron capture therapy in the treatment of locally recurred head and neck cancer. Int. J Radiat. Oncol. Biol. Phys. 69, 475-482.

Kankaanranta, L., Seppala, T., Koivunoro, H., Saarilahti, K., Atula, T., Collan, J., Salli, E. Kortesniemi, M., Uusi-Simola, J., Valimaki, P., Makitie, A., Seppanen, M., Minn, H., Revitzer, H., Kouri, M., Kotiluoto, P., Seren, T., Auterinen, I., Savolainen, S., Joensuu, H., 2012. Boron neutron capture therapy in the treatment of locally recurred head-and-neck cancer: final analysis of a phase I/II trial. Int. J. Radiat. Oncol. Biol. Phys. 82, e67-e75.

Kato, I., Fujita, Y., Maruhashi, A., Kumada, H., Ohmae, M., Kirihata, M., Imahori, Y, Suzuki, M., Sakrai, Y., Sumi, T., Iwai, S., Nakazawa, M., Murata, I., Miyamaru, H. Ono, K., 2009. Effectiveness of boron neutron capture therapy for recurrent head and neck malignancies. Appl. Radiat. Isot. 67, S37-S42.

Kimura, Y., Ariyoshi, Y., Shimahara, M., Miyatake, S., Kawabata, S., Ono, K., Suzuki, M., Maruhashi, A., 2009. Boron neutron capture therapy for recurrent oral cancer and metastasis of cervical lymph node. Appl. Radiat. Isot. 67, S47-S49.

Lartigau, E., Tresch, E., Thariat, J., Graff, P., Coche-Dequeant, B., Benezery, K. Sciappacasse, L., Degardin, M., Bondiau, P., Peiffert, D., Lefebvre, J., Lacornerie, T., Kramar, A., 2013. Multi institutional phase II study of concomitant stereotactic reirradiation and cetuximab for recurrent head and neck cancer. Radiother. Oncol. 109 (2), 281-285.

Lee, N., Chan, K., Bekelman, J.E., Zhung, J., Mechalakos, J., Narayana, A., Wolden, S., Venkatraman, E.S., Pfister, D., Kraus, D., Shah, J., Zelefsky, M.J., 2007. Salvage reirradiation for recurrent head and neck cancer. Int. J. Radiat. Oncol. Biol. Phys. $68,731-740$.

List, M.A., Siston, A., Haraf, D., Schumm, P., Kies, M., Stenson, K., Vokes, E.E., 1999. Quality of life and performance in advanced head and neck cancer patients on concomitant chemoradiotherapy: a prospective examination. J. Clin. Oncol. 17, 
1020-1028.

Mallick, S., Gandhi, A.K., Joshi, N.P., Pandit, S., Bhasker, S., Sharma, A., Thakar, A., Mohanti, B.K., 2014. Re-irradiation in head and neck cancers: an Indian tertiary cancer centre experience. The Journal of laryngology and otology 128 , 996-1002.

Riaz, N., Hong, J., Sherman, E., Morris, L., Fury, M., Ganly, I., wang, T., Shi, W. Wolden, S., Jackson, A., Wong, R., Zhang, Z., Rao, S., Lee, N., 2014. A nomogram to predict loco-regional control after re-irradiation for head and neck cancer. Radiother. Oncol. 111 (3), 382-387.

Roh, K.W., Jang, J.S., Kim, M.S., Sun, D.I., Kim, B.S., Jung, S.L., Kang, J.H., Yoo, E.J., Yoon, S.C., Jang, H.S., Chung, S.M., Kim, Y.S., 2009. Fractionated stereotactic radiotherapy as reirradiation for locally recurrent head and neck cancer. Int. J. Radiat. Oncol. Biol. Phys. 74, 1348-1355.

Stuschke, M., Kaiser, A., Abu-Jawad, J., Pottgen, C., Levegrun, S., Farr, J., 2013. Reirradiation of recurrent head and neck carcinomas: comparison of robust intensity modulated proton therapy treatment plans with helical tomotherapy. Radiat. Oncol. 8, 93.

Suzuki, M., Kato, I., Aihara, T., Hiratsuka, J., Yoshimura, K., Niimi, M., Kimura, Y., Ariyoshi, Y., Haginomori, S., Sakurai, Y., Kinashi, Y., Masunaga, S., Fukushima, M. Ono, K., Maruhashi, A., 2014. Boron neutron capture therapy outcomes for advanced or recurrent head and neck cancer. Journal of radiation research 55 , 146-153.

Tanvetyanon, T., Padhya, T., McCaffrey, J., Zhu, W., Boulware, D., Deconti, R., Trotti, A., 2009. Prognostic factors for survival after salvage reirradiation of head and neck cancer. J. Clin. Oncol. 27, 1983-1991.

Vermorken, J.B., Specenier, P., 2010. Optimal treatment for recurrent/metastatic head and neck cancer. Annals of oncology : official journal of the European Society for Medical Oncology / ESMO 21 Suppl 7, vii252-261.

Vermorken, J.B., Mesia, R., Rivera, F., Remenar, E., Kawecki, A., Rottey, S., Erfan, J. Zabolotnyy, D., Kienzer, H.R., Cupissol, D., Peyrade, F., Benasso, M., Vynnychenko, I., De Raucourt, D., Bokemeyer, C., Schueler, A., Amellal, N., Hitt, R., 2008. Platinum-based chemotherapy plus cetuximab in head and neck cancer. New Engl. J. Med. 359, 1116-1127.

Wang, L.W., Wang, S.J., Chu, P.Y., Ho, C.Y., Jiang, S.H., Liu, Y.W., Liu, Y.H., Liu, H.M., Peir, J.J., Chou, F.I., Yen, S.H., Lee, Y.L., Chang, C.W., Liu, C.S., Chen, Y.W., Ono, K., 2011. BNCT for locally recurrent head and neck cancer: preliminary clinical experience from a phase I/II trial at Tsing Hua Open-Pool Reactor. Appl. Radiat. Isot. 69, 1803-1806.

Weichselbaum, R.R., Beckett, M.A., Schwartz, J.L., Dritschilo, A., 1988. Radioresistant tumor cells are present in head and neck carcinomas that recur after radiotherapy. Int. J. Radiat. Oncol. Biol. Phys. 15, 575-579.

Zafereo, M.E., Hanasono, M.M., Rosenthal, D.I., Sturgis, E.M., Lewin, J.S., Roberts, D. B., Weber, R.S., 2009. The role of salvage surgery in patients with recurrent squamous cell carcinoma of the oropharynx. Cancer 115, 5723-5733.

Zimmermann, M., Zouhair, A., Azria, D., Ozsahin, M., 2006. The epidermal growth factor receptor (EGFR) in head and neck cancer: its role and treatment implications. Radiat. Oncol. 1, 11. 